\title{
Salamander-Derived, Human-Optimized nAG Protein Suppresses Collagen Synthesis and Increases Collagen Degradation in Primary Human Fibroblasts
}

\author{
Mohammad M. Al-Qattan, ${ }^{1,2}$ Medhat K. Shier, ${ }^{2}$ Mervat M. Abd-AlWahed, ${ }^{2}$ \\ Ola H. Mawlana, ${ }^{2}$ Mohammed S. El-Wetidy, ${ }^{2}$ Reginald S. Bagayawa, ${ }^{2}$ Hebatallah H. Ali, ${ }^{2}$ \\ May S. Al-Nbaheen, ${ }^{3}$ and Abdullah M. Aldahmash ${ }^{3,4}$ \\ ${ }^{1}$ Department of Surgery, King Saud University, Riyadh, Saudi Arabia \\ ${ }^{2}$ College of Medicine Research Center, King Saud University, Riyadh, Saudi Arabia \\ ${ }^{3}$ Stem Cell Unit, Department of Anatomy, College of Medicine, King Saud University, Riyadh, Saudi Arabia \\ ${ }^{4}$ Endocrine Research Laboratory (KMEB), Odense University Hospital \& University of Southern Denmark, \\ Odense, Denmark
}

Correspondence should be addressed to Mohammad M. Al-Qattan; moqattan@hotmail.com

Received 8 July 2013; Revised 15 September 2013; Accepted 19 September 2013

Academic Editor: Dong-Wook Han

Copyright (C) 2013 Mohammad M. Al-Qattan et al. This is an open access article distributed under the Creative Commons Attribution License, which permits unrestricted use, distribution, and reproduction in any medium, provided the original work is properly cited.

\begin{abstract}
Unlike humans, salamanders regrow their amputated limbs. Regeneration depends on the presence of regenerating axons which upregulate the expression of newt anterior gradient (nAG) protein. We had the hypothesis that nAG might have an inhibitory effect on collagen production since excessive collagen production results in scarring, which is a major enemy to regeneration. $n A G$ gene was designed, synthesized, and cloned. The cloned vector was then transfected into primary human fibroblasts. The results showed that the expression of nAG protein in primary human fibroblast cells suppresses the expression of collagen I and III, with or without TGF- $\beta 1$ stimulation. This suppression is due to a dual effect of nAG both by decreasing collagen synthesis and by increasing collagen degradation. Furthermore, nAG had an inhibitory effect on proliferation of transfected fibroblasts. It was concluded that nAG suppresses collagen through multiple effects.
\end{abstract}

\section{Introduction}

Tissue regeneration and wound healing are two different processes [1]. In regeneration, there is a suitable microenvironment for new tissue formation. Human liver, spleen, and skeletal muscle retain some ability to regenerate after damage $[2,3]$. Most of the new research in the field of regenerative medicine investigates the concept of stem cellbased tissue engineering approach for tissue regeneration $[4,5]$. In contrast, wound healing is a process of tissue repair mainly by fibrous tissue (collagen) formation [6]. Although healing by fibrous tissue is essential for "normal" tissue repair, excessive fibrosis is a harmful pathological process. Examples of pathological fibrotic conditions in humans include hypertrophic/keloid scars [7], Dupuytren contracture [8], idiopathic pulmonary fibrosis [9], myocardial interstitial fibrosis [10], and hepatic fibrosis [11].

Regrowth of amputated limbs of salamanders represents a unique example of tissue regeneration because there is regeneration of different types of tissues in the correct orientation. Therefore, the molecular events of the regenerating amputation stump are of interest in the field of biotechnology. The amputation stump of the salamander forms a blastema which is a mound of proliferating mesenchymal cells surrounded by wound epithelium. Regeneration depends on the presence of regenerating axons which upregulate the expression of newt anterior gradient (nAG) protein [12-15]. nAG protein expression by schwann cells of regenerating axons peaks at 
5-7 days postamputation. The nAG protein is released by schwann cells. At 10-12 days, the protein is also expressed in glands in the dermis underlying the wound epithelium [12-15]. The effect of $\mathrm{nAG}$ on dermal fibroblasts has not been previously investigated. We had the hypothesis that $\mathrm{nAG}$ might have an inhibitory effect of collagen production since excessive collagen production results in scarring, which is a major enemy to regeneration. The following experiments were conducted to test this hypothesis. We show that nAG suppresses collagen, both by decreasing collagen production and by increasing collagen degradation. We also show that nAG had an inhibitory effect on proliferation of transfected fibroblasts.

\section{Materials and Methods}

2.1. Ethics and Consent Statements. Primary human fibroblasts were isolated from infant human foreskin after obtaining written consent from the parents. Subjects (including human material or human data) reported in the current paper have been performed with the approval of the "College of Medicine Research Center, Deanship of Scientific Research, King Saud University, Riyadh, Saudi Arabia” Ethics Committee, in compliance with the Helsinki Declaration (http://www.wma.net/en/30publications/10policies/b3/ index.html).

2.2. nAG Gene Design and Synthesis. $\mathrm{nAG} \mathrm{mRNA}$ and protein sequences were obtained from NCBI nucleotide database. Notophthalmus viridescens anterior gradient protein (eastern newt) 2 mRNA is 820 bases and the protein is 166 amino acids. The first amino acid methionine (ATG) starts at nucleotide 66 of the mRNA and the last amino acid leucine (CTG) ends at nucleotide 563 of the mRNA sequence. This was confirmed by using translate utility of the bioinformatics tools available from DNA 2.0 company online. nAG amino acid sequence (166 AA) was entered into DNA 2.0 Gene Designer Software. The DNA sequence was depicted automatically by the software, giving different options of codon usage for many amino acids. Adjusting the software to homosapiens (25\% threshold), protein sequence is codon-optimized for efficient expression in human cells. Many amino acids are needed to be optimized for expression in human cells (more than 79\%) (see Supplementary Material available online at http://dx.doi.org/10.1155/2013/384091). Eukaryotic regulatory elements including transcription elements (enhancer, promoter, and polyadenylation signal sequence) and replication elements were not included in the design of $n A G$ gene. The only elements that were added are nAG mRNA $5^{\prime}$ UTR and $\mathrm{V} 5$ peptide for later detection of the protein using anti-V5 antibody. Transcription regulatory elements are supplied in the pJexpress 608 mammalian expression vector of DNA 2.0 Company (Figure 1(a)). nAG gene was also designed to contain two unique restriction enzymes Xhol and Not 1, for further use in molecular cloning. Xhol is designed to be upstream of the gene sequence and Not 1 to be downstream. Regarding GC content, Gene Designer software proposes only sequences with GC\% between $30 \%$ and $70 \%$. After optimization, the GC\% of the $n A G$ gene was $53.7 \%$. nAG plasmid integrity was confirmed by DNA electrophoresis showing the plasmid $(6.2 \mathrm{kbp})$ in two forms: circular uncut plasmid and linearized cut with XhoI (Figure 1(b)).

2.3. Transformation and Preservation of $n A G$-pJexpress 608. Confirmation of nAG-pJexpress 608 (synthesized by DNA 2.0, USA) was done by performing $1 \%$ agarose gel electrophoresis for both circular plasmid and digested plasmid with Xhol enzyme (Figure 1). Propagation of plasmid was performed by transformation of $\mathrm{nAG}$ plasmid into competent DH5 $\alpha$ bacterial cells, prepared in our laboratory by using $\mathrm{CaCL}_{2}$ method according to the protocol of Current Protocols in Molecular Biology. Transformation was done with $10 \mathrm{ng}$ of nAG-pJexpress 608 added to $100 \mu \mathrm{L}$ of competent DH5 $\alpha$ heat shocked for $45 \mathrm{sec}$ at $42^{\circ} \mathrm{C}$ followed by incubation on ice for 10 minutes, then selection of transformed bacterial cells on Ampicillin LB agar plates, and finally preservation of bacterial cells in glycerol stock at $-80^{\circ} \mathrm{C}$.

2.4. Fibroblasts Isolation and Culture. Dermal specimen was washed repeatedly with phosphate-buffered saline (PBS) (Gibco, Carlsbad, California, USA) with a combination of $1 \%$ penicillin and streptomycin sulfate (Gibco) and minced aseptically into approximately $1 \mathrm{~mm}^{3}$ pieces. The specimens were then placed in $10 \mathrm{~cm}$ culture plate with $5 \mathrm{~mL}$ of culture medium (Dulbecco Modified Eagle Medium (DMEM), $10 \%$ fetal bovine serum, $1 \%$ nonessential amino acid, and $1 \%$ penicillin-streptomycin sulfate) (Gibco) at $37^{\circ} \mathrm{C}$ in a humidified $5 \% \mathrm{CO}_{2}$ incubator. After 5 days the medium was repeatedly changed every $48 \mathrm{~h}$ and examined under microscope untill fibroblasts were seen growing outwards from the explanted tissue. Then the tissue was removed and sufficient outgrowth of fibroblasts was subcultured and propagated. For testing collagen synthesis experiments, $150 \mu \mathrm{g} / \mathrm{mL}$ L-ascorbic acid (Bio Basic Inc., Ontario, Canada) and TGF- $\beta 1$ (Santa Cruz, Texas, USA) $3 \mathrm{ng} / \mathrm{mL}$ (for collagen I) and $10 \mathrm{ng} / \mathrm{mL}$ (for collagen III) were added for culture medium.

2.5. Plasmid Transfection (Lipofection and Electroporation) into Primary Human Fibroblast. Lipofection for nAG plasmid was carried out using Lipofectamine 2000 (Invitrogen, Carlsbad, California, USA); cells were seeded in a complete medium without antibiotic until they reach $80-90 \%$ confluence in $6 \mathrm{~cm}$ plates. Lipofection was done using 1:3 DNA : Lipofectamine ratio. DNA : Lipofectamine complexes were removed after 5 hours and replaced with complete medium.

Electroporation for $\mathrm{nAG}$ plasmid was performed into primary human fibroblasts by aliquoting $800 \mu \mathrm{L}$ of cell suspension in electroporation buffer $(150 \mathrm{mOsmol} / \mathrm{kg})$ and $20 \mu \mathrm{g}$ of $\mathrm{nAG}$ plasmid into a cuvette with $4 \mathrm{~mm}$ gap width. Multiporator (Eppendorf, Hamburg, Germany) was adjusted on eukaryotes mode, $800 \mathrm{~V}$ with time constant $60 \mu \mathrm{sec}$ and the cells received two pulses. After $10 \mathrm{~min}$, the cells were plated in $6 \mathrm{~cm}$ plates for western blot assay and in 8 chambers slide (Millipore, Billerica, MA, USA) for immunofluorescence assay. 


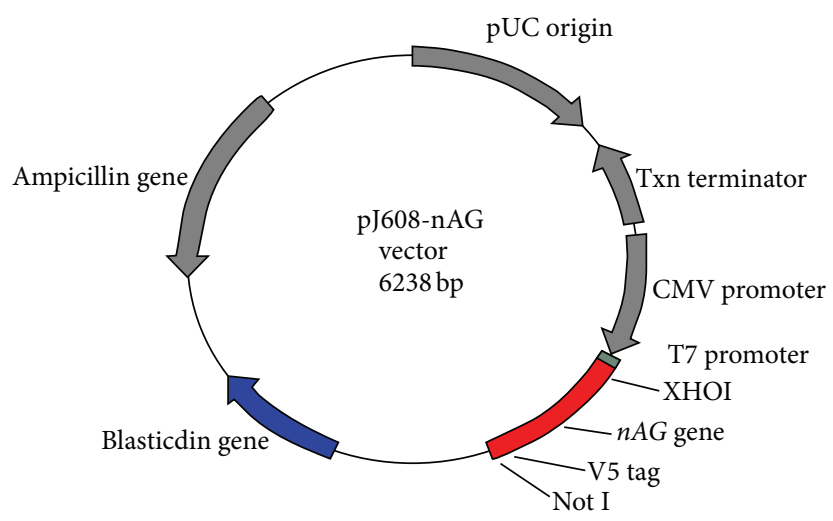

(a)

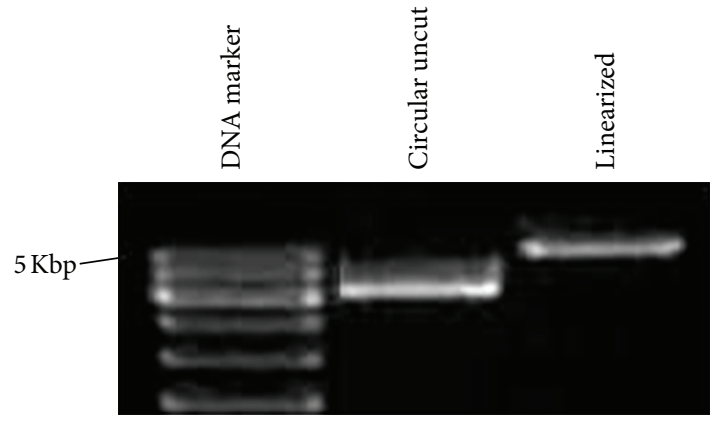

(b)

FIGURE 1: Identification of $n A G$ gene in mammalian expression plasmid nAG-pJexpress pJ608. (a) The plasmid contains $n A G$ gene, optimized for human cell expression and tagged with V5 peptide for further identification. The gene is surrounded by two restriction sites: XhoI and Not I. (b) nAG plasmid integrity was confirmed by DNA electrophoresis showing the plasmid in two forms: circular uncut plasmid and linearized cut with XhoI.

2.6. Western Blotting. After $24 \mathrm{~h}$ and $48 \mathrm{~h}$ of transfection, cells were scrabed and lysed using RIPA cocktail (RIPA buffer, PMS, sodium orthovanadate, and protease inhibitor) (Santa Cruz). Cell suspensions were centrifuged at $14.000 \mathrm{rpm}$ for 15 minutes at $4^{\circ} \mathrm{C}$. Total cellular protein concentrations were measured by Bradford reagent (Sigma, Louis, USA). Equal amounts of proteins were separated on SDS polyacrylamide gel $12 \%$ for nAG separation (18.9 kd) and $7.5 \%$ SDS polyacrylamide gel for collagen I (90 kd) and collagen III $(140 \mathrm{kd})$ at $100 \mathrm{~V}$ for $2 \mathrm{~h}$. Proteins were transferred into PVDF membranes (Millipore) using $100 \mathrm{~V}$ for $1.5 \mathrm{~h}$. After the transfer, membranes were blocked by $10 \%$ blocking buffer Blotto (Santa Cruz) dissolved in TBST for $1 \mathrm{hr}$ at room temperature. The membranes then were incubated with primary antibodies (V5-probe, Santa Cruz) dissolved in blocking buffer $(1: 600)$ for nAG detection, (COL1A1, Santa Cruz) (1:200) for collagen I detection, (COL3A1, Santa Cruz) $(1: 200)$ for collagen III detection, and $\beta$-actin (sc-69879, Santa Cruz) (1:1000). After wash, the membranes were incubated with the appropriate HRP-conjugated secondary antibody (Santa Cruz) dissolved in blocking buffer (1:10000) for $1 \mathrm{~h}$ at room temperature. After washing the immunoblots were visualized by ECL kit (Amersham, Buckinghamshire, UK) and the bands' densities were quantified using Quantity One software (Bio-Rad). Results were expressed as ratio of band density to $\beta$-actin.

2.7. Immunofluorescence. Transfected cells were plated at density of $8 \times 10^{4}$ cells/well, and after 48 hours, medium was removed and cells were fixed and permeabilized by $2 \%$ PFA (Santa Cruz)/0.1\% Triton x-100 (LKB Bromma, Sweden) in PBS for 30 minutes. After washing, cells were blocked by $1 \%$ BSA (Santa Cruz) in PBST (1\% Triton X-100 in PBS) for 30 minutes. The cells were incubated with primary antibodies V5-probe (sc-58052, Santa Cruz) for nAG detection, COL1A1 (sc-28657, Santa Cruz) for collagen I detection, and COL3A1
(sc-8780-R, Santa Cruz) for collagen III detection using 1:50 dilution in blocking buffer for 1 hour at room temperature. After washing, bound primary antibodies were detected by incubation with the appropriate FITC-conjugated secondary antibodies (Santa Cruz) 1:100 dilution for 1 hour in a dark chamber and then examined under fluorescence microscope (Olympus, Center Valley, PA, USA).

\subsection{Proliferation Activity of Nontransfected and nAG Trans-} fected Fibroblasts (BrdU Incorporation Assay). The cells were cultured in 96-well plates at a density of 8000 cells/well in complete growth media. After 24 and 48 hours, proliferation of non-transfected and nAG transfected fibroblasts was assayed by incorporation of pyrimidine analogue 5bromo- 2 -deoxyuridine (BrdU) using ELISA, BrdU (Roche, Mannheim, Germany). Cells were labeled using $10 \mu \mathrm{M}$ BrdU per well and incubated for $2 \mathrm{~h}$ at $37^{\circ} \mathrm{C}$ in a humidified atmosphere. After fixation, the cells were incubated with the antiBrdU-POD antibody for 90 minutes at room temperature. After the removal of the antibody conjugate, the cells were washed and the substrate solution was added. The reaction product was quantified by measuring the absorbance using a Microplate Reader Synergy 2 (BioTek, Winooski, Vermont, USA) at $370 \mathrm{~nm}$.

\subsection{Collagen Synthesis}

2.9.1. RNA Extraction and Quantitative Real-Time PCR Analysis. Total RNA was isolated both from fibroblasts transfected with nAG plasmid and from normal fibroblasts by using the RNeasy protect mini kit (Qiagen, Dusseldorf, Germany) according to the manufacturer's instructions. $100 \mathrm{ng}$ of total RNA was reverse-transcribed and target genes expression was measured in multiplex, one-step RT-PCR using rotorgene multiplex PCR kit (Qiagen, Dusseldorf, Germany) according to the manufacturer's instructions. Specific primers 
TABLE 1: Primers and probes used for quantitative real-time PCR.

\begin{tabular}{|c|c|c|c|}
\hline Gene & Gene accession number & Primer, probe sequence & Product size \\
\hline \multirow{3}{*}{ Procollagen I } & \multirow{3}{*}{ AC number NM_000089.3 } & Forward: TGG ATT GAC CCT AAC CAA GGA TGC & \multirow{3}{*}{145} \\
\hline & & Reverse: AGA CGT GTT TCT TGT CCT TGG AGC & \\
\hline & & Probe: ACT GGC GAA ACC TGT ATC CGG GCC CAA CCT & \\
\hline \multirow{3}{*}{ Procollagen III } & \multirow{3}{*}{ AC number NM_000090.3 } & Forward: AGT CCT GGT GGT AAA GGC GAA ATG & \multirow{3}{*}{150} \\
\hline & & Reverse: TTT GGC ACC ATT CTT ACC AGG CTC & \\
\hline & & Probe: AAT GGT GCT CCT GGA CTG CGA GGT GGT GCA & \\
\hline \multirow{3}{*}{$\beta$-actin } & \multirow{3}{*}{ AC number NM_001101.3 } & Forward: ACCGAGCGCGGCTACAG & \multirow{3}{*}{60} \\
\hline & & Reverse: CTTAATGTCACGCACGATTTCC & \\
\hline & & Probe: TTCACCACCACGGCCGAGC & \\
\hline
\end{tabular}

and TaqMan probes were designed using Integrated DNA Technologies software (San Diego, California, USA). The TaqMan probes had a fluorescent reporter dye (FAM, HEX, or ROX) attached to the $5^{\prime}$ end, while the Quencher BHQ1 (for FAM and HEX) and BHQ2 (for ROX) were attached to the $3^{\prime}$ end. Sequences of the primers and probes used are listed in Table 1 . Thermal cycling conditions were as follows: an initial reverse transcription step for $15 \mathrm{~min}$ at $50^{\circ} \mathrm{C}$, incubation at $95^{\circ} \mathrm{C}$ for $5 \mathrm{~min}$ to activate hot start DNA polymerase, and then 40 cycles at $95^{\circ} \mathrm{C}$ for $15 \mathrm{sec}$ and $60^{\circ} \mathrm{C}$ for $15 \mathrm{sec}$. Acquiring of the fluorescent signal on green, yellow, and orange channels occurs in the annealing/extension step. Using $\beta$-actin as a normalizing gene and procollagen I and procollagen III in normal fibroblasts as calibrators, relative quantification of procollagen I and procollagen III expression levels in $\mathrm{nAG}$ transfected fibroblasts was obtained using $\Delta \Delta$ ct relative quantification method in Rotor-gene $\mathrm{Q}$ 5 plex software (Qiagen). The relative mRNA expressions in transfected and control fibroblasts were determined by three independent quantitative real-time PCR experiments.

\subsection{Collagen Degradation}

2.10.1. Gelatin Zymography for MMP-2 Detection. MMP proteins with gelatinolytic activity were determined in conditioned media deprived from serum for 24 hours for normal fibroblasts and fibroblasts transfected with $\mathrm{nAG}$ plasmid and treated with $3 \mathrm{ng} / \mathrm{mL}$ TGF- $\beta 1.20 \mathrm{uL}$ of undiluted cell culture supernatant was mixed with an equal volume of nonreducing SDS sample buffer, using MMP-2 (R\&D Systems, Minneapolis, USA) as positive control. Samples were loaded on $7.5 \%$ separating SDS polyacrylamide gel with $0.1 \%$ gelatin (LOBA Chemie, Mumbai, India) as substrate and electrophoresis was done at constant voltage of $100 \mathrm{~V}$ for 2 hours. The gels were then washed in renaturing buffer (2.5\% Triton X-100) for 1 hour to promote recovery of protease activity before incubation in zymogram development buffer at $37^{\circ} \mathrm{C}$. The gels were stained with Coomassie blue R-250. After destaining, clear digested regions representing MMP gelatinolytic action appeared against blue background. The optical density and area of the bands were compared using densitometry in gel documentation instrument (Bio-Rad, Berkeley, USA).
2.10.2. Assessment of Pro-MMP-1 Level Using ELISA. ELISA was performed using Quantikine Human Pro-MMP-1 ELISA (R\&D Systems, Minneapolis, USA). Protocol was done as per the manufacturer directions. Standards and samples (culture media of non transfected fibroblasts, fibroblasts transfected with $\mathrm{nAG}$ plasmid, and fibroblasts treated with $1 \mu \mathrm{M}$ recombinant $n A G$ ) were added and incubated in microplate wells precoated with anti-pro-MMP-1 mouse monoclonal antibody. After washing away unbound substances, horseradish peroxidase linked monoclonal antibody specific for proMMP-1 is added to the wells. Following a wash and addition of substrate solution, the reaction was stopped and the resultant colour was read at $450 \mathrm{~nm}$ using microplate reader Synergy 2 (BioTek, Winooski, Vermont, USA).

2.11. Statistical Analysis. Data were expressed as means \pm standard deviation (SD) and analyzed by one-way ANOVA test and $P$ value less than 0.05 was considered statistically significant. The statistical difference was determined using Student' $t$-test for independent groups. All experiments were performed at least three replicates.

\section{Results}

3.1. nAG Expression in Primary Fibroblast Cells. Western blot assay confirmed $\mathrm{nAG}$ protein expression in human fibroblasts showing that a specific $\mathrm{nAG}$ protein band corresponds to $18.9 \mathrm{kd}, \mathrm{nAG}$ protein expression appeared after 24 hours, and the amount of protein expression increased after 48 hours as shown in the increasing band density (Figure 2(a)).

Immunofluorescence staining with primary antibody V5probe showed $\mathrm{nAG}$ protein expression in primary human fibroblast and fluorescent green color was evident after 24 hours of pJ608-nAG plasmid transfection (Figure 2(b)).

\subsection{Proliferation Activity in Nontransfected and nAG Trans-} fected Fibroblasts. BrdU incorporation ELISA assay was performed to test effect of $\mathrm{nAG}$ on fibroblasts' proliferation. The results showed the inhibitory effect of $\mathrm{nAG}$ on fibroblasts proliferation. Compared to control (non-transfected fibroblasts), 


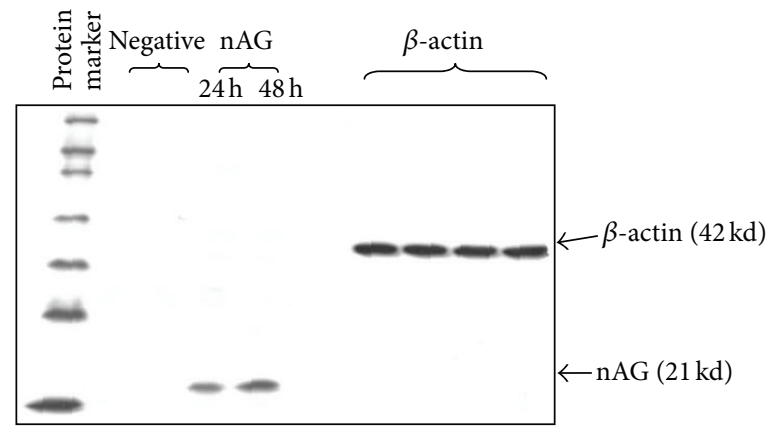

(a)

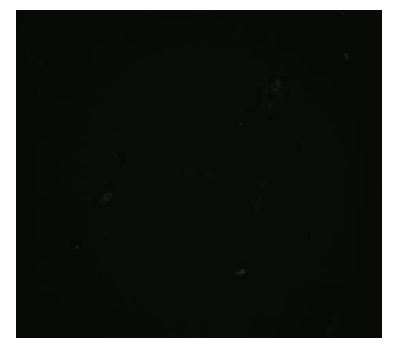

Negative control

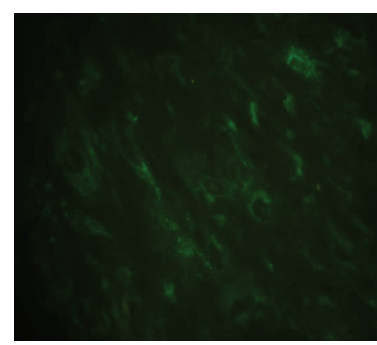

Fibroblasts transfected with $\mathrm{nAG}$

(b)

FIGURE 2: Western blot assay (a) and immunofluorescence (b) experiments showing nAG protein expression in primary human fibroblasts. (a) Western blot was performed after $24 \mathrm{~h}$ and $48 \mathrm{~h}$ of transfection. The cells were lysed by using RIPA cocktail; proteins were separated on $12 \%$ SDS polyacrylamide gel. The primary antibody V5 probe and HRP-conjugated secondary antibody were used for $\mathrm{nAG}$ protein detection in the following lanes: nontransfected fibroblasts (negative control 1), fibroblasts with nAG plasmid without lipofection (negative control 2), nAG transfected fibroblasts tested after $24 \mathrm{~h}$ and $\mathrm{nAG}$ transfected fibroblasts tested after $48 \mathrm{~h}$. (b) Immunofluorescence assay was performed after $48 \mathrm{~h}$ of transfection and cells were fixed and permeabilized by $2 \%$ PFA/0.1\% Triton $\mathrm{x}-100$. V5 probe was used as primary antibody and green fluorescence was detected by using FITC-conjugated secondary antibody. Compare nAG protein expression in nontransfected fibroblasts (negative control) to $\mathrm{nAG}$ expression in transfected fibroblasts (magnification 40x).

there was inhibition in proliferation by $47 \%$ decrease $(\mathrm{SD} \pm$ 8.39) $(P<0.0001)$ after $24 \mathrm{~h}$ of transfection and by $42 \%$ decrease $(\mathrm{SD} \pm 6.096)(P<0.0001)$ after $48 \mathrm{~h}$ in nAG transfected fibroblasts (Figure 3).

\subsection{Collagen Expression in Fibroblasts Expressing $n A G$ with} or without TGF- $\beta 1$. Western blot assay showed collagen I expression in untreated control fibroblasts (Lane 1, Figure 4(a)) and control fibroblasts treated with TGF- $\beta 1$ (Lane 3, Figure 4(a)). Transfected fibroblasts without treatment with TGF- $\beta 1$ (Lane 2, Figure 4(a)) showed decreased collagen I expression compared to untreated control fibroblasts. With TGF- $\beta 1$ treatment, there was also decreased collagen I expression in transfected fibroblasts (Lane 4, Figure 4(a)) compared to treated control fibroblasts. The effect on collagen I involved both full length protein $(129 \mathrm{KD})$ and the degradation fragment (30 KD) (Figure 4(a)).

Western blot assay showed collagen III expression in untreated control fibroblasts (Lane 1, Figure 4(b)) and control fibroblasts treated with TGF- $\beta 1$ (Lane 2, Figure 4(b)). Transfected fibroblasts without treatment with TGF- $\beta 1$ (Lane 3 , Figure 4(b)) showed decreased collagen III expression compared to untreated control fibroblasts. With TGF- $\beta 1$ treatment, there was also decreased collagen III expression in transfected fibroblasts (Lane 4, Figure 4(b)) compared to treated control fibroblasts. The effect on collagen III was complete suppression of the full length protein $(140 \mathrm{KD})$. This indicated that $\mathrm{nAG}$ protein expression in human fibroblast cells has an inhibitory effect on the expression of collagen I and III and that the inhibitory effect of nAG is dominant over the TGF- $\beta 1$ effect. Furthermore, the inhibitory effect of nAG protein was more pronounced on collagen III than collagen I.

Immunofluorescence (Figure 5) confirmed the results of the western blot assay. There was decreased collagen I

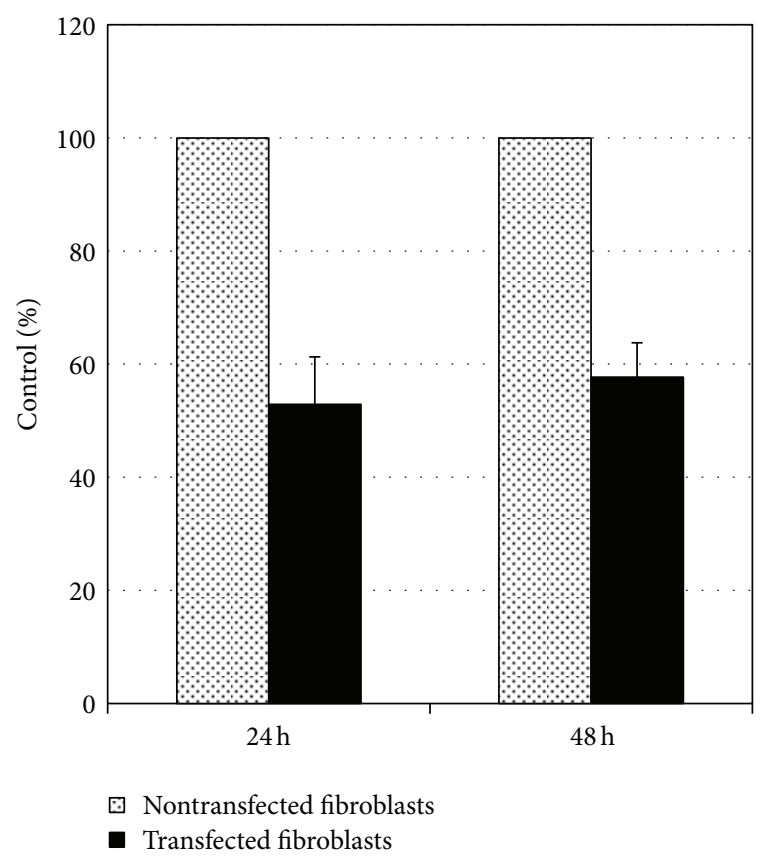

FIGURE 3: BrdU incorporation ELISA assay for assessment of proliferation activity in nontransfected and nAG transfected fibroblasts. The cells were cultured in 96-well plates at a density of 8000 cells/well. 24 and 48 hours after lipofection, cells were assayed for proliferation by measuring BrdU incorporation during DNA synthesis in proliferating cells. The results showed the inhibitory effect of nAG on fibroblasts proliferation, after $24 \mathrm{~h}$ by $47 \%$ decrease $(P<0.0001)$ and after $48 \mathrm{~h}$ by $42 \%$ decrease $(P<0.0001)$ in proliferation in $\mathrm{nAG}$ transfected fibroblasts compared to nontransfected fibroblasts.

expression in transfected fibroblasts (with and without TGF$\beta 1$ ) compared to controls (Figure 5(a)); and there was complete collagen III suppression in transfected fibroblasts (with 


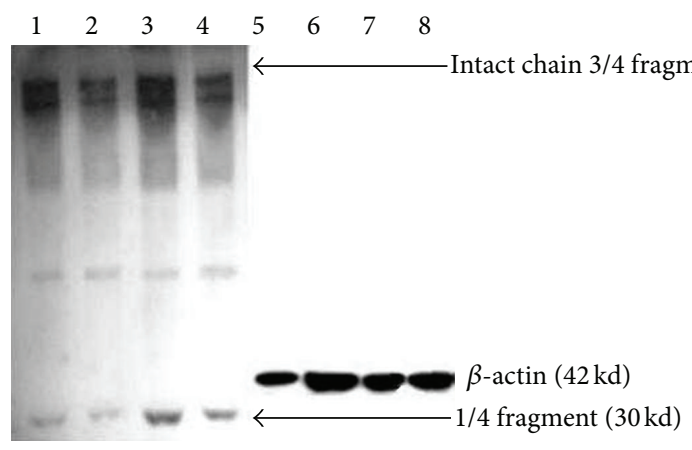

(a)

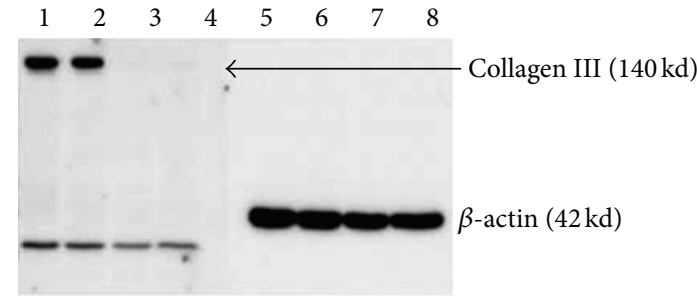

(b)

FIGURE 4: Western blot experiment for detection of collagen I (a) and collagen III (b) expression in primary human fibroblasts transfected with nAG plasmid with or without treatment with TGF- $\beta 1$. Cells were lysed after 48 hours using RIPA cocktail. Proteins were separated on 7.5\% SDS polyacrylamide gel and detected by using COL1A1 (primary antibody for collagen I), COL3A1 (primary antibody for collagen III), and HRP-conjugated secondary antibody and the immunoblots were visualized by using ECL kit. (a) Collagen I detection: lane-1: collagen I expression in non-transfected fibroblasts without any treatment (control 1), lane-2: collagen I expression in fibroblasts transfected with nAG plasmid, lane-3: collagen I expression in non-transfected fibroblasts treated with TGF- $\beta 1$ (control 2), lane-4: collagen I expression in fibroblasts transfected with nAG plasmid and treated with TGF- $\beta 1$, and lanes-5-8: internal control $\beta$-actin. (b) Collagen III detection: lane-1: collagen III expression in non-transfected fibroblasts without any treatment (control 1), lane-2: collagen III expression in non-transfected fibroblasts with TGF- $\beta 1$ treatment (control 2), lane-3: collagen III expression in fibroblasts transfected with nAG plasmid, lane-4: collagen III expression in fibroblasts transfected with nAG plasmid and treated with TGF- $\beta 1$, and lanes- 5 to 8 are internal control $\beta$-actin.
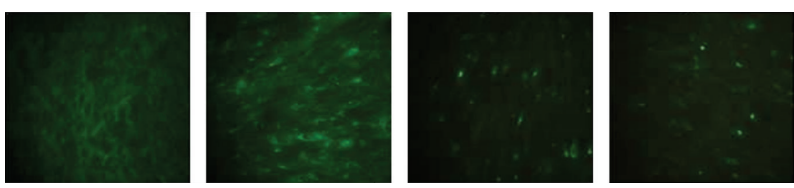

Fibroblasts-control cells Fibroblasts with TGF- $\beta 1$ Fibroblasts with nAG Fibroblasts with nAG and TGF- $\beta 1$

(a)
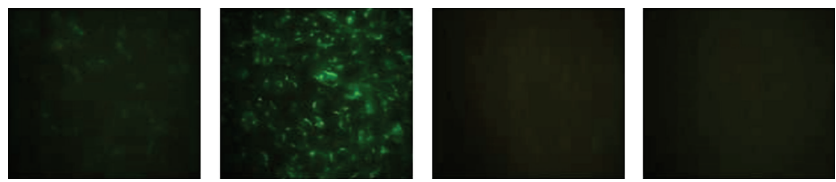

Fibroblasts-control cells Fibroblasts with TGF- $\beta 1$

(b)

FIGURE 5: Immunofluorescence experiment showing effect of nAG on collagen I (a) and collagen III (b) expression in primary human fibroblasts with or without TGF- $\beta 1$ treatment. After $48 \mathrm{~h}$ of transfection, immunoflourescence was performed by using COL1A1, COL3A1 (primary antibodies), and FITC-conjugated secondary antibody. (a) Immunofluorescence staining of type I collagen is in the following order: control fibroblasts (complete medium $+150 \mu \mathrm{g} / \mathrm{mL}$ L-ascorbic acid), control fibroblasts treated with $3 \mathrm{ng} / \mathrm{mL}$ TGF- $\beta 1$, fibroblasts with nAG plasmid, and fibroblasts with nAG plasmid and treated with $3 \mathrm{ng} / \mathrm{mL}$ TGF- $\beta 1$ (magnification $40 \mathrm{x}$ ). Note the suppressive effect of $\mathrm{nAG}$ on collagen I. (b) Immunofluorescence staining of type III collagen is in the following order: control fibroblasts (complete medium $+150 \mu \mathrm{g} / \mathrm{mL}$ L-ascorbic acid), control fibroblasts treated with $10 \mathrm{ng} / \mathrm{mL}$ TGF- $\beta 1$, fibroblasts with $\mathrm{nAG}$ plasmid, and fibroblasts with nAG plasmid and treated with $10 \mathrm{ng} / \mathrm{mL}$ TGF- $\beta 1$ (magnification 40x). Note the complete suppressive effect of $\mathrm{nAG}$ on collagen III.

and without TGF- $\beta 1$ ) compared to controls (Figure 5(b)). This also confirmed that the suppressive effect of $n A G$ was more pronounced on collagen III expression than collagen I.

3.4. Quantitative Real-Time PCR Analysis. Relative quantification of mRNA expression levels in non-transfected and $\mathrm{nAG}$ transfected fibroblasts was performed to quantify the effect of $\mathrm{nAG}$ on procollagen I and procollagen III mRNA synthesis. The results confirmed that $\mathrm{nAG}$ protein suppresses procollagen I mRNA expression by $55 \%$ decrease (SD \pm 0.028) $(P<0.001)$ and suppresses procollagen III mRNA expression by $95 \%$ decrease $(\mathrm{SD} \pm 0.011)(P<0.0001)$ in transfected fibroblasts than in non-transfected cells (Figure 6). The data represents the mean of three independent experiments. 


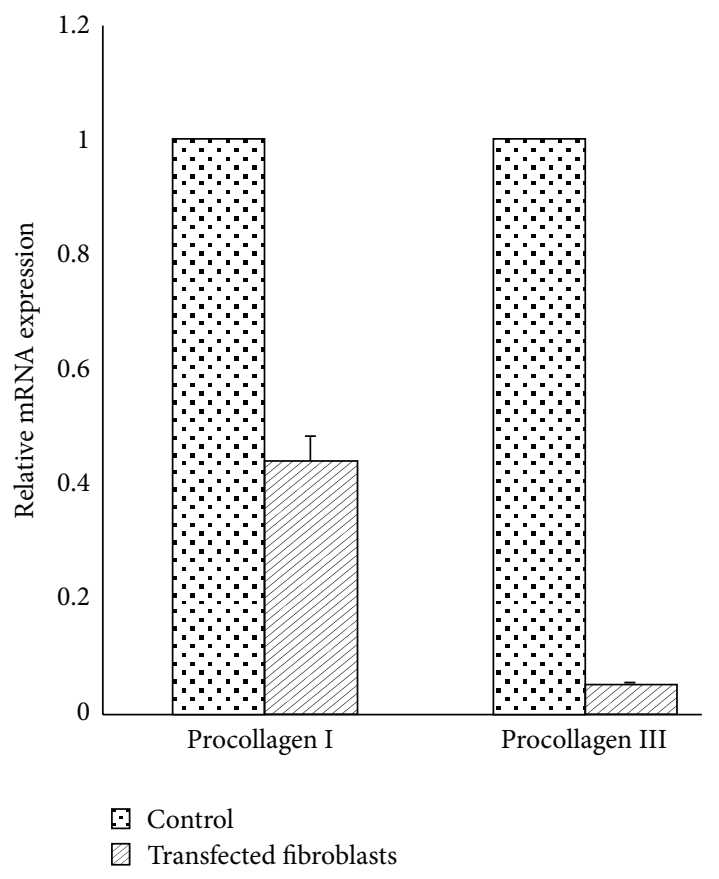

FIgURE 6: Quantitative real-time PCR (RT-PCR) measuring relative mRNA expressions level of procollagen I and procollagen III in nontransfected and nAG transfected primary human fibroblasts. $100 \mathrm{ng}$ of total RNA was reverse-transcribed and target genes expression was measured in multiplex, one-step RT-PCR by using TaqMan probes with (FAM, HEX, or ROX) reporter dyes and (BHQ1 or BHQ2) quencher. To estimate effect of $n A G$ on collagen I and collagen III synthesis, the relative mRNA expressions were related to the reference gene, $\beta$-actin. The relative expression of procollagen I was 55\% decrease $(P<0.001)$ and procollagen III was $95 \%$ decrease $(P<0.0001)$ in nAG transfected fibroblasts compared to non-transfected fibroblasts. The data represents the mean of three independent experiments.

3.5. Gelatin Zymography for MMP-2 Detection. We performed gelatin zymography to determine the effect of $\mathrm{nAG}$ on MMP-2 gelatinase activity (which degrades gelatin and collagen) in non-transfected (control cells) and nAG transfected fibroblasts (Figure $7(\mathrm{a})$ ). There were $37 \%$ increase in pro-MMP-2 $(\mathrm{SD} \pm 15.33)(P=0.019)$ and $85 \%$ increase in the active form of MMP-2 $(\mathrm{SD} \pm 2.38)(P=0.001)$ in $\mathrm{nAG}$ transfected fibroblasts compared to non-transfected fibroblasts (Figure 7(b)). This indicated that $\mathrm{nAG}$ increases collagen degradation.

3.6. Pro-MMP-1 Level Using ELISA. Assessment of proMMP-1 protein level in culture media of non-transfected fibroblasts (control), nAG transfected fibroblasts, and fibroblasts treated with $\mathrm{nAG}$ recombinant protein showing that there was 53 fold increase in pro-MMP-1 expression levels $(\mathrm{SD} \pm 257.9)(P=0.004)$ in culture media of nAG transfected fibroblasts compared to non-transfected fibroblasts, and there was 4 -fold increase in pro-MMP-1 expression levels (SD \pm 18.03) $(P<0.0001)$ in fibroblasts treated with recombinant nAG compared to untreated cells (Figure 7(c)). Since MMP-1 collagenase activity has the ability to cleave the native helical structure of interstitial collagen I, II, and III, these results indicated that $\mathrm{nAG}$ increases collagen degradation.

\section{Discussion}

The current work opens new insights on the effects of nAG on collagen. We have designed a $n A G$ gene that is suitable for human cells. We demonstrated the successful expression of nAG in human fibroblasts and the suppressive effect of nAG on the expression of collagen I and III. No previous studies have investigated the effect of $\mathrm{nAG}$ on collagen and hence we are unable to compare our results to others.

Although collagen formation is essential for tissue healing, excessive collagen is pathological [16]. The TGF- $\beta$ pathway mediates both normal wound healing and scar formation [17]. Following the activation of the receptors by TGF- $\beta 1$, SMADs 2 and 3 are phosphorylated and the activated SMADs bind to SMAD-4. This process is controlled by the inhibitory SMAD-7. The activated SMAD complex will then translocate from the cytoplasm into the nucleus to regulate gene expression for collagen synthesis and degradation [1820].

Pathological fibrosis is characterized by abnormally high levels of TGF- $\beta$, increased proliferation of fibroblasts, increased collagen synthesis, decreased collagen degradation, and the appearance of myofibroblasts [21-25].

Type I collagen is the most abundant and is synthesized in response to injury [26]. Type III collagen is also synthesized in response to dermal injury. In normal wound healing, the newly formed type III collagen is gradually replaced by collagen I to maintain the normal collagen I to collagen III ratio in the dermis. In contrast, there is persistent expression of excessive type III collagen in pathological fibrosis [27].

Matrix metalloproteinase (MMPs) are considered to be the most important enzymes mediating collagen degradation [28]. The active forms of MMPs are formed when the prodomains are removed by autolytic cleavage or by other proteases $[29,30]$. Normally, there is a balance between the MMPs and their inhibitors known as tissue inhibitors of matrix metalloproteinase (TIMPs). In pathological fibrosis this balance is disturbed with marked decrease in MMPs. The imbalance leads to increase collagen accumulation.

Our results showed that $\mathrm{nAG}$ has an inhibitory effect on collagen expression by both decrease in collagen production and increasing collagen degradation. This dual effect is interesting because pathological fibrosis is characterized by both increased collagen production and decreased collagen degradation. Another interesting finding is the fact that the suppressive effect of $\mathrm{nAG}$ on collagen is evident with or without TGF- $\beta 1$ stimulation. This dominance over the TGF$\beta 1$ effect is important because excessive TGF- $\beta 1$ stimulation is a feature of several fibrotic conditions [31]. Finally, our work demonstrated that the suppressive effect of $n A G$ on collagen production is more pronounced on collagen III compared to collagen $\mathrm{I}$. This is also interesting because collagen III overproduction is the hallmark of pathological fibrosis [32]. 


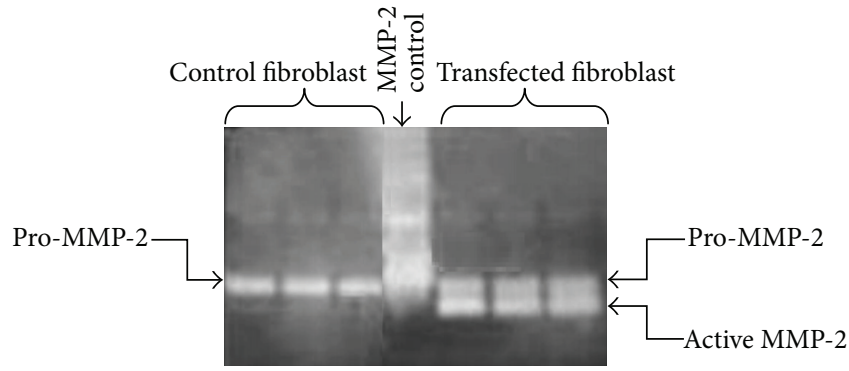

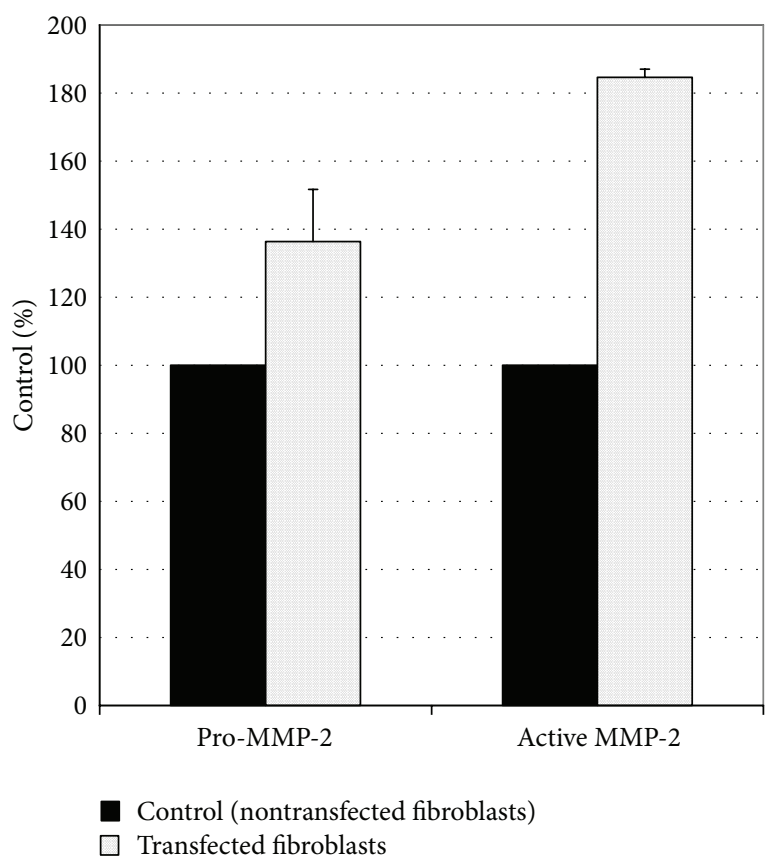

(b)

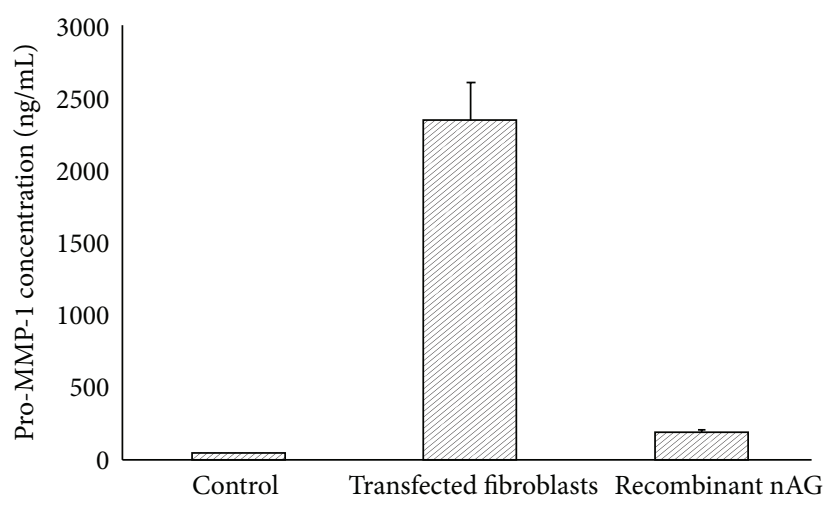

(c)

FIGURE 7: Determination of pro-MMP-2 and active MMP-2 by gelatin zymography ((a) and (b)) and pro-MMP-1 by ELISA (c) in nontransfected and nAG transfected fibroblasts. (a) Gelatin zymography: media were harvested after two days of transfection of nAG plasmid into fibroblasts and non-transfected fibroblasts (both were treated with $3 \mathrm{ng} / \mathrm{mL}$ TGF- $\beta 1$ ). The first three lanes were different fractions of the same sample of non-transfected fibroblasts, the fourth lane was MMP-2 (positive control), and the last three lanes were samples of nAG transfected fibroblasts. (b) Densitometry analysis for gelatin zymography: density of each band was measured by using densitometry tool in Gel documentation software. There was 37\% increase in pro-MMP-2 $(P<0.019)$ and $85 \%$ increase in active MMP-2 $(P<0.001)$ in transfected fibroblasts compared to non-transfected fibroblasts. (c) ELISA assay for pro-MMP-1 measurement: after two days of transfection. Media were harvested for measurement of pro-MMP-1 in non-transfected fibroblasts (control), nAG transfected fibroblasts, and fibroblasts treated with recombinant nAG. The level of pro-MMP-1 was 53-fold increase in transfected fibroblast compared to non-transfected fibroblasts $(P<0.004)$ and it was 4 -fold increase in nAG-treated fibroblasts compared to non-transfected fibroblasts $(P<0.0001)$.

\section{Conclusion}

nAG suppresses collagen expression. This suppressive effect is mediated through multiple actions including decreased fibroblast proliferation, decreased collagen I and III production, and increased collagen degradation. Furthermore, the effects of $\mathrm{nAG}$ are dominant over TGF- $\beta$ stimulation.

\section{Conflict of Interests}

The authors declare that they have no conflict of interests.

\section{Authors' Contribution}

The senior author (Mohammad M. Al-Qattan) had the original idea and the hypothesis that $\mathrm{nAG}$ may have an 
inhibitory effect on collagen. The senior author also designed the experiment. The rest of the authors performed the lab work.

\section{Acknowledgment}

This project was funded by College of Medicine Research Center, Deanship of Scientific Research, King Saud University, Riyadh, Saudi Arabia.

\section{References}

[1] S. F. Williams, S. Risk, and D. P. Martin, "Poly-4 hydroxybutyrate $(\mathrm{P} 4 \mathrm{HB})$ : a new generation of resorbable medical devices for tissure repair and regeneration," Biomedizinische Technik, vol. 58, no. 5, pp. 439-452, 2013.

[2] M. Saclier, S. Cuvellier, M. Magnan, R. Mounier, and B. Chazaud, "Monocyte/macrophage interactions with myogenic precursor cells during skeletal muscle regeneration," FEBS Journal, vol. 280, no. 17, pp. 4118-4130, 2013.

[3] G. Millet, S. Truant, E. Leteurtre et al., "Volumetric analysis of remnant liver regeneration after major hepatectomy in bevacizumab-treated patients: a case-matched study in 82 patients," Annals of Surgery, vol. 256, no. 5, pp. 755-761, 2012.

[4] P. T. Brown, A. M. Handorf, W. B. Jeon, and W. J. Li, "Stem cell-based tissue engineering approaches for musculoskeletal regeneration," Current Pharmaceutical Design, vol. 19, no. 19, pp. 3429-3445, 2013.

[5] R. Lakshmanan, U. M. Kirishran, and S. Sethuraman, "Polymeric scaffold aided stem cell therapeutics for cardiac muscle repair and regeneration," Macromolecular Bioscience, vol. 13, no. 9, pp. 1119-1134, 2013.

[6] A. J. Singer and R. A. F. Clark, "Cutaneous wound healing," The New England Journal of Medicine, vol. 341, no. 10, pp. 738-746, 1999.

[7] T. A. Mustoe, R. D. Cooter, M. H. Gold et al., "International clinical recommendations on scar management," Plastic and Reconstructive Surgery, vol. 110, no. 2, pp. 560-571, 2002.

[8] M. M. Al-Qattan, "Factors in the pathogenesis of Dupuytren's contracture," Journal of Hand Surgery, vol. 31, no. 9, pp. 1527$1534,2006$.

[9] M. Couluris, B. W. Kinder, P. Xu et al., "Treatment of idiopathic pulmonary fibrosis with losartan: a pilot project," Lung, vol. 190, no. 5, pp. 523-527, 2012.

[10] Z. Dai, T. Aoki, Y. Fukumoto, and H. Shimokaw, "Coronary perivascular fibrosis is associated with impairment of coronary blood flow in patients with non-ischemic heart failure," Journal of Cardiology, vol. 60, no. 5, pp. 416-421, 2012.

[11] T. Liu, X. Wang, M. A. Karsdal et al., "Molecular serum markers of liver fibrosis," Biomarker Insights, vol. 7, pp. 105-117, 2012.

[12] A. Kumar, J. W. Godwin, P. B. Gates, A. A. Garza-Garcia, and J. P. Brockes, "Molecular basis for the nerve dependence of limb regeneration in an adult vertebrate," Science, vol. 318, no. 5851, pp. 772-777, 2007.

[13] A. Kumar, G. Nevill, J. P. Brockes, and A. Forge, "A comparative study of gland cells implicated in the nerve dependence of salamander limb regeneration," Journal of Anatomy, vol. 217, no. 1, pp. 16-25, 2010.

[14] J. P. Brockes, "Amphibian limb regeneration: rebuilding a complex structure," Science, vol. 276, no. 5309, pp. 81-87, 1997.

[15] J. F. Brockes and A. Kumar, "Appendage regeneration in adult vertebrates and implications for regenerative medicine," Science, vol. 310, no. 5756, pp. 1919-1923, 2005.
[16] S. Aarabi, M. T. Longaker, and G. C. Gurtner, "Hypertrophic scar formation following burns and trauma: new approaches to treatment," PLoS Medicine, vol. 4, no. 9, pp. 1464-1470, 2007.

[17] A. Leask and D. J. Abraham, "TGF- $\beta$ signaling and the fibrotic response," FASEB Journal, vol. 18, no. 7, pp. 816-827, 2004.

[18] A. J. Singer, S. S. Huang, J. S. Huang et al., "A novel TGFbeta antagonist speeds reepithelialization and reduces scarring of partial thickness porcine burns," Journal of Burn Care and Research, vol. 30, no. 2, pp. 329-334, 2009.

[19] R. Wieser, J. L. Wrana, and J. Massague, "GS domain mutations that constitutively activate T $\beta$ R-I, the downstream signaling component in the TGF- $\beta$ receptor complex," EMBO Journal, vol. 14, no. 10, pp. 2199-2208, 1995.

[20] X. Wang, Y. Qian, R. Jin et al., "Effects of TRAP-1-like protein (TLP) gene on collagen synthesis induced by TGF- $\beta / \mathrm{Smad}$ signaling in human dermal fibroblasts," PLoS ONE, vol. 8, no. 2, Article ID e55899, 2013.

[21] T. L. Adair-Kirk and R. M. Senior, "Fragments of extracellular matrix as mediators of inflammation," International Journal of Biochemistry and Cell Biology, vol. 40, no. 6-7, pp. 1101-1110, 2008.

[22] K. E. Kadler, C. Baldock, J. Bella, and R. P. Boot-Handford, "Collagens at a glance," Journal of Cell Science, vol. 120, no. 12, pp. 1955-1958, 2007.

[23] G. C. Blobe, W. P. Schiemann, and H. F. Lodish, "Role of transforming growth factor $\beta$ in human disease," The New England Journal of Medicine, vol. 342, no. 18, pp. 1350-1358, 2000.

[24] G. J. Prud'homme, "Pathobiology of transforming growth factor $\beta$ in cancer, fibrosis and immunologic disease, and therapeutic considerations," Laboratory Investigation, vol. 87, no. 11, pp. 1077-1091, 2007.

[25] M.-J. Goumans, Z. Liu, and P. Ten Dijke, “TGF- $\beta$ signaling in vascular biology and dysfunction," Cell Research, vol. 19, no. 1, pp. 116-127, 2009.

[26] K. Gelse, E. Pöschl, and T. Aigner, "Collagens-structure, function, and biosynthesis," Advanced Drug Delivery Reviews, vol. 55, no. 12, pp. 1531-1546, 2003.

[27] G. C. Gurtner, S. Werner, Y. Barrandon, and M. T. Longaker, "Review article wound repair and regeneration," Nature, vol. 453, no. 7193, pp. 314-321, 2008.

[28] K. Asanuma, R. Magid, C. Johnson, R. M. Nerem, and Z. S. Galis, "Uniaxial strain upregulates matrix-degrading enzymes produced by human vascular smooth muscle cells," American Journal of Physiology-Heart and Circulatory Physiology, vol. 284, no. 5, pp. H1778-H1784, 2003.

[29] I. Tchetverikov, N. Verzijl, T. W. J. Huizinga, J. M. TeKoppele, R. Hanemaaijer, and J. DeGroot, "Active MMPs captured by alpha2Macroglobulin as a marker of disease activity in rheumatoid arthritis," Clinical and Experimental Rheumatology, vol. 21, no. 6, pp. 711-718, 2003.

[30] R. Visse and H. Nagase, "Matrix metalloproteinases and tissue inhibitors of metalloproteinases: structure, function, and biochemistry," Circulation Research, vol. 92, no. 8, pp. 827-839, 2003.

[31] Z. Lin, S. Zhong, D. Liu, Y. Mao, and P. Ning, "Effect of tetrandrine on the TGF- $\beta$-induced smad signal transduction pathway in human hypertrophic scar fibroblasts in vitro," Burns, vol. 38, no. 3, pp. 404-413, 2012.

[32] G. V. Oliveira, H. K. Hawkins, D. Chinkes et al., "Hypertrophic versus non hypertrophic scars compared by immunohistochemistry and laser confocal microscopy: type i and III collagens," International Wound Journal, vol. 6, no. 6, pp. 445452, 2009. 

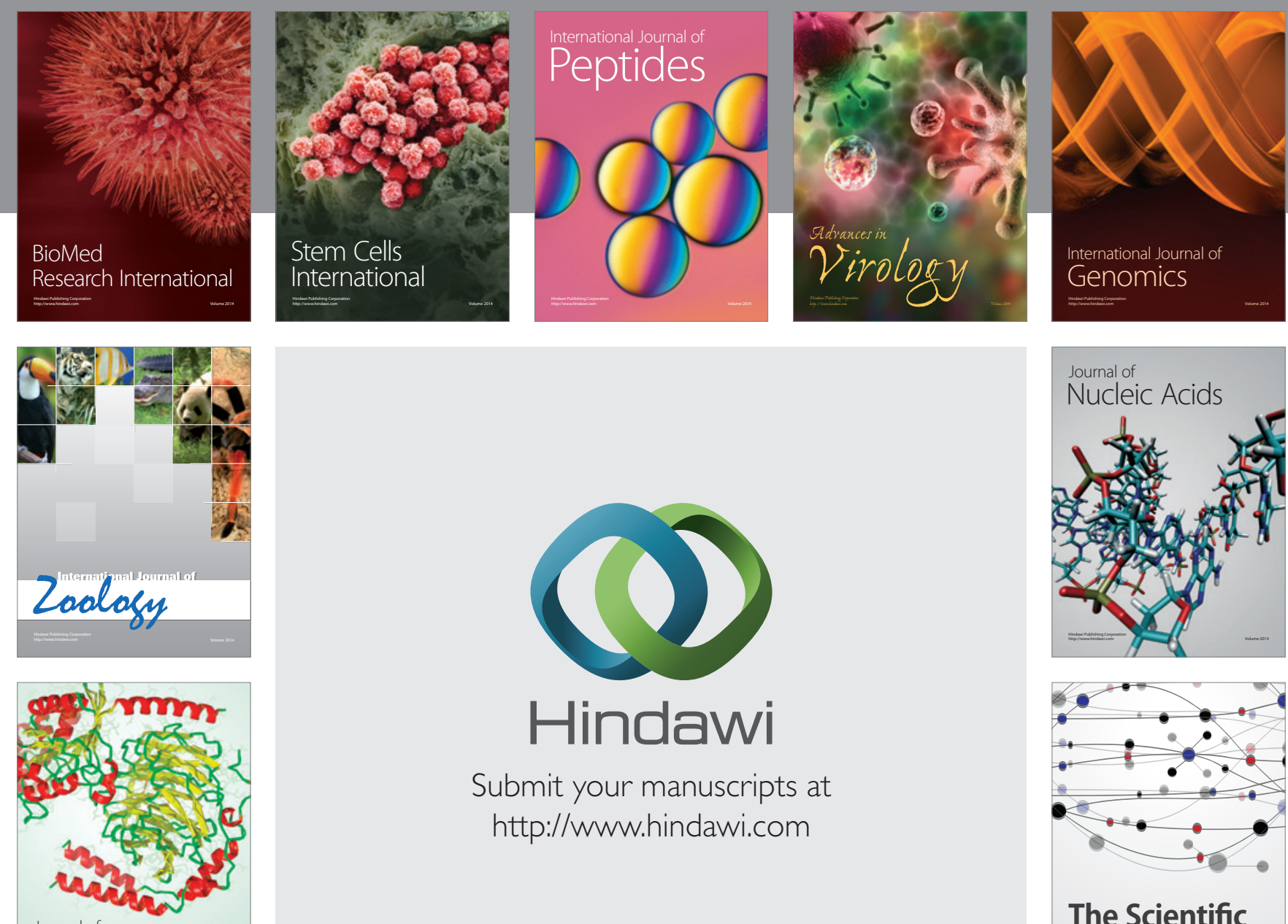

Submit your manuscripts at

http://www.hindawi.com

Journal of
Signal Transduction
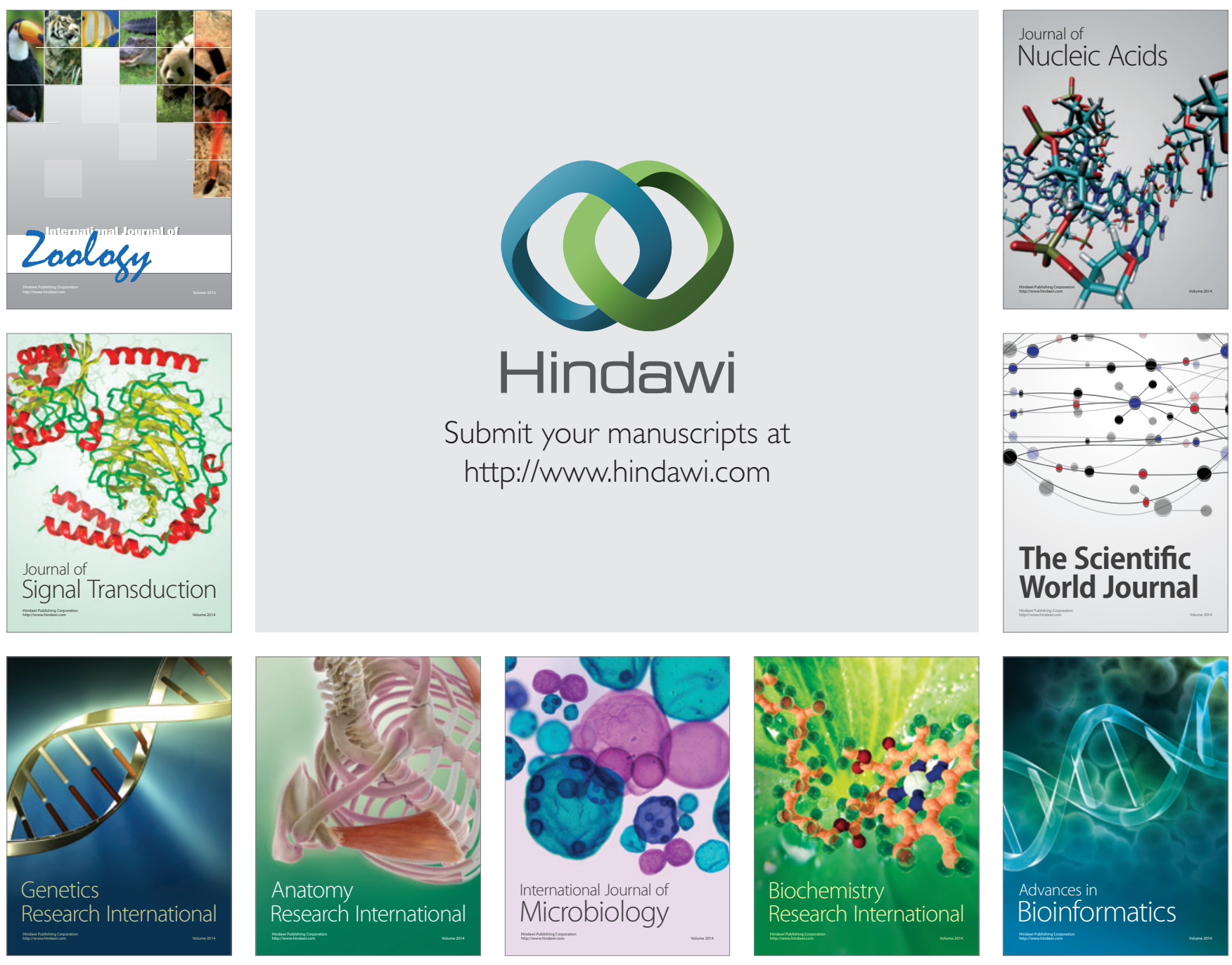

The Scientific World Journal
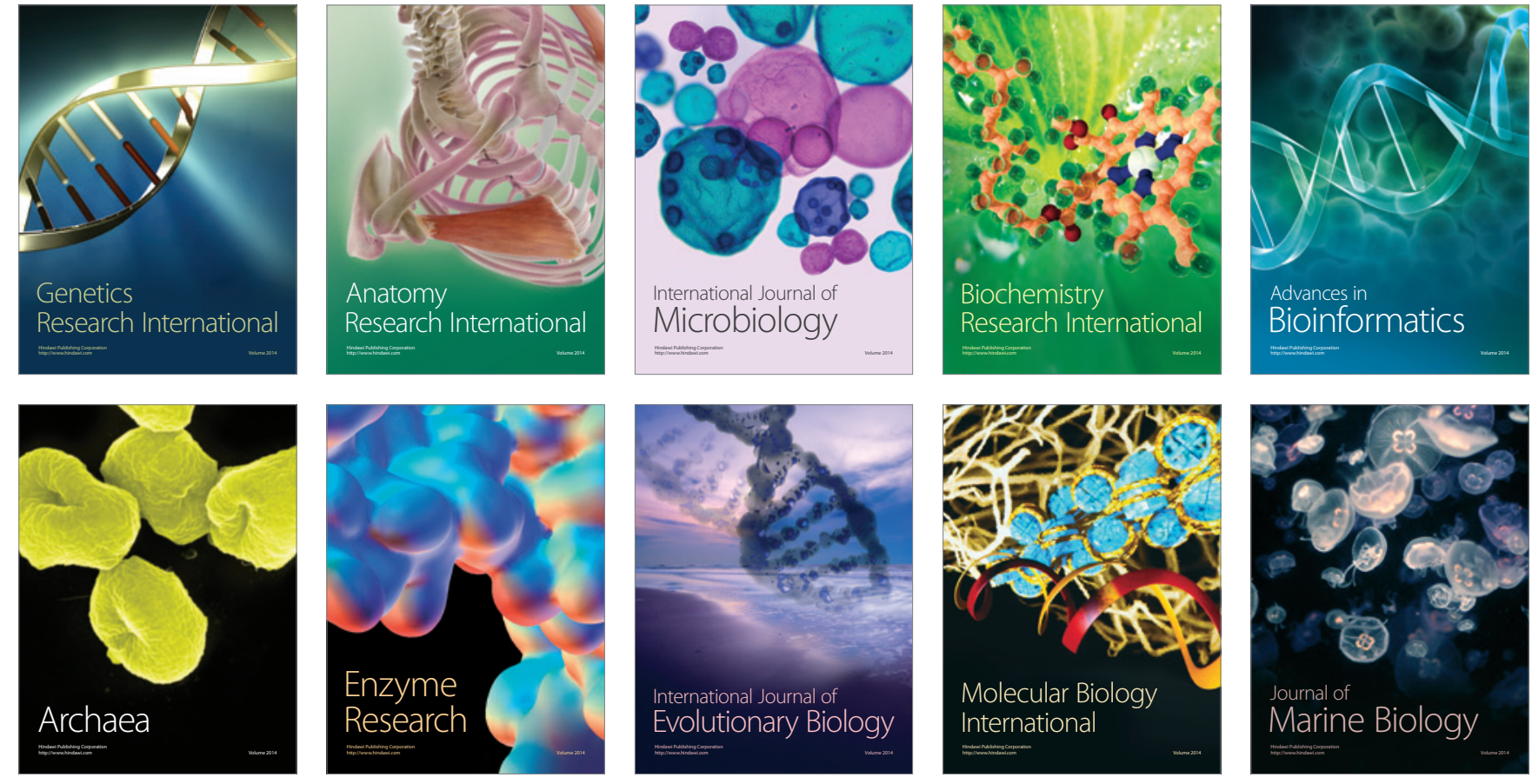